\title{
RECONSTRUCTION OF A BROADBAND SPECTRUM OF ALFVÉNIC FLUCTUATIONS
}

\author{
Adolfo F Viñas ${ }^{1}$, Pablo S. Moya ${ }^{1,2}$, Jaime A. Araneda ${ }^{3}$, and Yana G. Maneva Mand $^{1,2}$ \\ ${ }^{1}$ NASA Goddard Space Flight Center, Heliophysics Science Division, Geospace Physics Laboratory, \\ Mail Code 673, Greenbelt, MD 20771, USA; adolfo.vinas@nasa.gov \\ 2 Department of Physics, Catholic University of America, Washington, DC 20064, USA \\ ${ }^{3}$ Departamento de Física, Universidad de Concepción Facultad de Ciencias Físicas y Matemáticas, Casilla 160-C, Chile \\ Received 2013 August 22; accepted 2014 March 13; published 2014 April 21
}

\begin{abstract}
Alfvénic fluctuations in the solar wind exhibit a high degree of velocities and magnetic field correlations consistent with Alfvén waves propagating away and toward the Sun. Two remarkable properties of these fluctuations are the tendencies to have either positive or negative magnetic helicity $\left(-1 \leqslant \sigma_{m} \leqslant+1\right)$ associated with either left- or righttopological handedness of the fluctuations and to have a constant magnetic field magnitude. This paper provides, for the first time, a theoretical framework for reconstructing both the magnetic and velocity field fluctuations with a divergence-free magnetic field, with any specified power spectral index and normalized magnetic- and crosshelicity spectrum field fluctuations for any plasma species. The spectrum is constructed in the Fourier domain by imposing two conditions-a divergence-free magnetic field and the preservation of the sense of magnetic helicity in both spaces-as well as using Parseval's theorem for the conservation of energy between configuration and Fourier spaces. Applications to the one-dimensional spatial Alfvénic propagation are presented. The theoretical construction is in agreement with typical time series and power spectra properties observed in the solar wind. The theoretical ideas presented in this spectral reconstruction provide a foundation for more realistic simulations of plasma waves, solar wind turbulence, and the propagation of energetic particles in such fluctuating fields.
\end{abstract}

Key words: magnetic fields - methods: analytical - turbulence - waves

Online-only material: color figures

\section{INTRODUCTION}

Alfvénic fluctuations are ubiquitous and represent the dominant component of low-frequency magnetohydrodynamic (MHD) turbulence in the solar wind, and they are believed to play an important role in the heating and acceleration of solar wind ions (Marsch 2006; Bruno \& Carbone 2013). Moreover, an Alfvénic fluctuation propagating in a homogeneous plasma represents an exact solution of the nonlinear MHD equations provided that the total magnetic field intensity is constant.

Since the first in situ observations of Alfvén waves in the solar wind by Belcher \& Davis (1971), the problem of reconstruction of an Alfvénic spectrum and its correlation with velocity fluctuations have been a challenging paradigm in MHD turbulence. Although the reality of these fluctuations is that they are three-dimensional and broadband, the reconstruction of these waves even for a one-dimensional field-aligned propagation has resisted proper solution. Barnes (1981) treated the fluctuating fields as a "random walk" on a sphere and showed important properties that relates the alignment of the minimumvariance propagation direction of these waves to the mean field direction as well as their quasi-circular topology and the near constancy of the field magnitude. Further studies of these waves (e.g., Matthaeus \& Goldstein 1982) emphasized the importance of their topological structure by means of the magnetic- and cross-helicities in describing these fluctuations in solar wind turbulence. However, no reconstruction of the field fluctuations constrained to all these physically based characteristics has been suggested. Roberts (2012) has shown that even for a onedimension construction, which only imposes the divergencefree $(\nabla \cdot \mathbf{B}=\mathbf{0})$ condition, does not satisfactorily reproduce (see Figure 1 of his paper) realistic fluctuations as observed in the solar wind. His observationally motivated optimized approach minimizing the magnetic field magnitude condition has produced better results. The method uses a least-squares scheme to get the phases that minimizes the field magnitude variance. The model reasonably reproduces some of the solar wind magnetic fluctuation properties such as the alignment of the minimum variance of the field with the mean field direction and a magnitude near constant. However, the Roberts (2012) model is not motivated by first principles and it does not consider the magnetic helicity and cross-helicity conditions required to describe Alfvénic fluctuations (Matthaeus \& Goldstein 1982). Instead, it is mostly guided by the observations.

Reconstruction of either monochromatic or broadband spectrum of Alfvénic fluctuations is important for many studies based upon the linearized MHD description to fully nonlinear numerical simulations of the evolution of these waves in a plasma medium (Cohen \& Dewar 1974; Cohen 1975; Hoshino \& Goldstein 1989; Umeki \& Terasawa 1992; Ghosh et al. 1994; Agim et al. 1995; Araneda et al. 2008). The construction of a broadband spectrum of Alfvénic fluctuations as input for theoretical and computational studies constrained by observations has been an important aspect to physically based models addressing MHD turbulence (Podesta \& Gary 2011; He et al. 2011, 2012; Wicks et al. 2012; Bruno \& Carbone 2013), parametric instabilities (Matteini et al. 2010a, 2010b; Maneva et al. 2013), and nonlinear evolution (Moya et al. 2012) during the last few decades. Within the context of broadband spectrum and parametric instabilities, Malara \& Velli (1996) studied largeamplitude nonmonochromatic linearized ideal MHD Alfvén waves using a numerical approach to obtain a description of the fluctuating magnetic field components as a function of wavelength to generate a spectral representation of the fluctuations and to show that such a constant magnitude of the B spectrum remained unstable to parametric decay.

In this paper, we provide an alternate approach to the reconstruction of an initial self-consistent broadband spectrum 


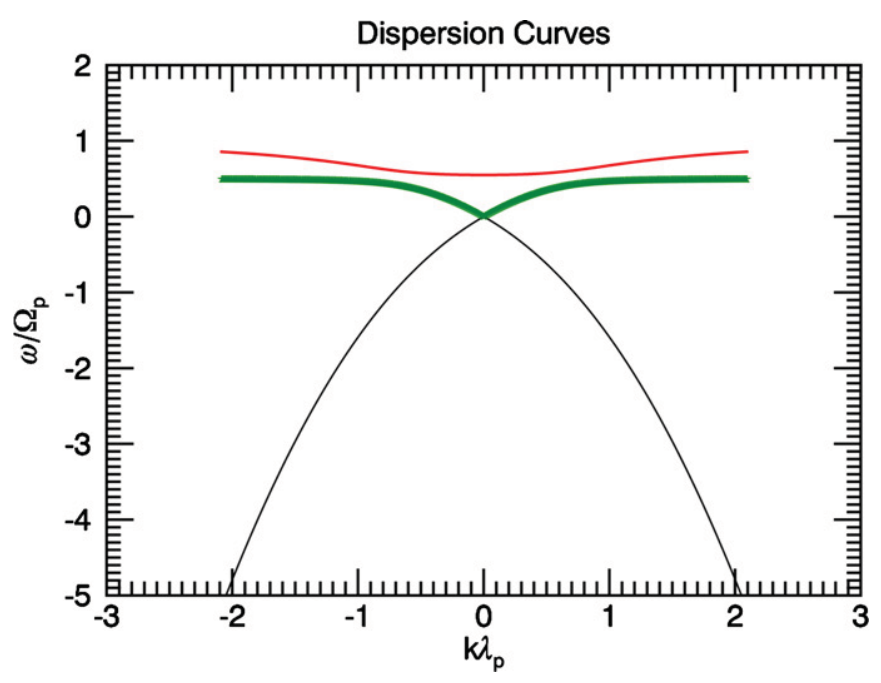

Figure 1. Dispersion relation for Alfvén waves in a cold electron, proton and alphas plasma. Red and green curves are left-hand polarized proton and alphas cyclotron branches, black curves are the right-hand polarized fast magnetosonic modes. Frequencies and wavenumbers are in units of proton gyrofrequency and inertial length, respectively.

(A color version of this figure is available in the online journal.)

of Alfvénic fluctuations that is simpler, physically motivated, and guided and constrained by the observations. The spectral theory is formulated completely in the Fourier domain, which is based on the linear superposition of wave modes. We have assumed that the fluctuations propagate in one spatial dimension and periodic boundary conditions are imposed. We impose two physically based conditions: a divergence-free magnetic field $(\nabla \cdot \mathbf{B}=0)$; and the preservation of the magnetic helicity $H_{\mathrm{mk}}$ between both domains, which can be either right- or lefthanded, and their sense of orientation and magnitude can be constrained by theory or observations (Podesta \& Gary 2011; He et al. 2011, 2012; Wicks et al. 2012). We further make use of Parseval's theorem that imposes energy density conservation between the Fourier and configuration domains. This final condition is necessary if we want to characterize the wave modes as Alfvénic fluctuations. The magnetic field magnitude $|\mathbf{B}|$ is nonuniform due to the fluctuations produced at all scales by beating between individual mode components, which are themselves circularly polarized and therefore have a constant magnitude of $\mathbf{B}$ associated with them. Since our model focused on other Alfvénic characteristics (i.e., spectral energy, magnetic helicity, cross-helicity, etc.) describing a turbulence broadband spectrum, we do not impose the constraint of magnetic field constancy, but provide an alternate approach using some of the other conditions that have been measured in the solar wind. Nonetheless, a comparison of our results with the magnetic field constancy test is provided to mimic fluctuations with rms amplitudes similar to those observed in the solar wind. In a study of parametric decay instability by incoherent Alfvénic waves, Umeki \& Terasawa (1992) provided a spectral representation of an initial spectrum. In their model of the spectral reconstruction, they assumed random phases, a single helicity, a total maximum power amplitude, and a spectral profile. These conditions are similar to those used in our model reconstruction. The main difference between the two models is that the spectral Fourier amplitude coefficient of each wave mode in our model is explicitly determined in terms of the conditions of divergencefree field, the spectral representation of the magnetic helicity, and the total magnetic energy. Furthermore, our model allows for constant or random magnetic helicity for each wave mode and the cross-helicity representation.

This paper is organized as follows. In the next section, we present our model to reconstruct an Alfvénic broadband spectrum, including the details needed to relate magnetic field fluctuations to the plasma properties. Section 3 shows applications of the model for two-species solar-wind-like plasmas. In Section 4, we summarize our results.

\section{RECONSTRUCTION OF A BROADBAND SPECTRUM}

Consider an initial magnetic field given by $\mathrm{B}(x)=\boldsymbol{B}_{0}+$ $\boldsymbol{B}_{\perp}(x)$, where $\mathbf{B}_{0}=B_{0} \hat{\boldsymbol{x}}$ is a constant ambient magnetic field and $\mathbf{B}_{\perp}(x)$ represents the transverse wave field propagating along the $x$ axis. The transverse fluctuating part can be described in Fourier space as a superposition of finite amplitude, circularly polarized, left- and right-handed waves according to

$$
\begin{aligned}
\mathrm{B}_{\perp}(x)= & R e\left[\sum _ { k > 0 } \left(C_{k}^{L} \exp \left(i \varphi_{k}\right)(\hat{\mathbf{y}}+i \hat{\mathbf{z}})\right.\right. \\
& \left.\left.+C_{k}^{R} \exp \left(i \psi_{k}\right)(\hat{\mathbf{y}}-i \hat{\mathbf{z}})\right) \exp (i k x) .\right]
\end{aligned}
$$

Here the constant pairs $\left(C_{k}^{L}, C_{k}^{R}\right)$ and $\left(\varphi_{k}, \psi_{k}\right)$ are the Fourier amplitude coefficients for left- (L) and right-handed (R) waves and the random phases for each wave mode, respectively. The divergence-free condition is fulfilled by construction. However, without knowledge of the Fourier coefficients and the random phases, we cannot represent the transverse broadband spectrum of the fluctuations. We shall assume that the phases $\left(\varphi_{k}, \psi_{k}\right)$ are random, between $\pm(0,2 \pi)$. By imposing the aforementioned physical conditions in the fluctuation energy density and their magnetic helicity, we can obtain relations that allow us to determine the Fourier coefficients $\left(C_{k}^{L}, C_{k}^{R}\right)$ in the Fourier domain. This is an important aspect of our model since the Fourier coefficients can be fully obtained in terms of the normalized spectral magnetic helicity $\sigma_{\mathrm{mk}}$ for each Fourier mode $k$, the power spectral energy density profile $\left(\left|\mathbf{B}_{k}\right|^{2}=\mathbf{B}_{k} \cdot \mathbf{B}_{k}^{*}\right)$, and the maximum rms amplitude $\left(\eta=\delta B / B_{0}\right)$ of the fluctuations, all of which are observable quantities that can be modeled.

In components, Equation (1) reads:

$$
\begin{aligned}
& B_{y}(x)=2 \operatorname{Re}\left[\sum_{k>0} B_{\mathrm{yk}} \exp (i k x)\right] \\
& B_{z}(x)=2 \operatorname{Re}\left[\sum_{k>0} B_{\mathrm{zk}} \exp (i k x)\right],
\end{aligned}
$$

where we define

$$
\begin{gathered}
B_{\mathrm{yk}}=\frac{1}{2}\left(C_{k}^{L} \exp \left(i \varphi_{k}\right)+C_{k}^{R} \exp \left(i \psi_{k}\right)\right) \\
B_{\mathrm{zk}}=\frac{i}{2}\left(C_{k}^{L} \exp \left(i \varphi_{k}\right)-C_{k}^{R} \exp \left(i \psi_{k}\right)\right) .
\end{gathered}
$$

We make use of Parseval's theorem condition (energy conservation in the Fourier domain), which is imposed by defining the total transverse magnetic energy per unit length in terms of the power spectral profile as

$$
\begin{aligned}
E & =\frac{1}{2} \int\left|\mathbf{B}_{\perp}(x)\right|^{2} d x \equiv 2 \operatorname{Re}\left(\sum_{k>0}\left|B_{k}\right|^{2}\right) \\
& \equiv 2 \operatorname{Re}\left(\sum_{k>0}\left[\left|B_{\mathrm{yk}}\right|^{2}+\left|B_{\mathrm{zk}}\right|^{2}\right]\right) .
\end{aligned}
$$


After some algebraic manipulation, it can be shown that the relationship between the power spectral profile and the Fourier coefficients is given by

$$
\left|B_{k}\right|^{2}=\left(\left|C_{k}^{L}\right|^{2}+\left|C_{k}^{R}\right|^{2}\right)
$$

Let us now assume the ansatz $\left|B_{k}\right|^{2}=C^{2} k^{-\alpha}$, which resembles a typical power spectrum in the solar wind where $\alpha$ is the spectral index and $C$ is a constant that can be easily defined in terms of the maximum spectral wave amplitude (rms) $\eta$ as

$$
\begin{aligned}
C^{2} & =\frac{\eta^{2} B_{0}^{2}}{2 \sum_{k>0} k^{-\alpha}} \quad \text { and } \quad \eta^{2}=\left(2 \sum_{k>0}\left|B_{k}\right|^{2}\right) / B_{0}^{2} \\
& =\left(\delta B / B_{0}\right)^{2} .
\end{aligned}
$$

The total transverse magnetic energy is conserved and can be expressed in terms of the Fourier coefficients and $\eta$. However, since there are two Fourier coefficients for each mode, we require another condition that can be easily obtained from the spectral representation of the magnetic helicity.

The magnetic helicity is defined as $H_{m}=\langle\mathbf{A} \cdot \mathbf{B}\rangle / 2$, where the operator $\langle\cdots\rangle$ represents the space average of the dot product of the vector potential $\mathrm{A}$ and the magnetic field $\boldsymbol{B}$ or the autocorrelation. The magnetic helicity $H_{m}$ is a measure of the spatial handedness of the magnetic field topology. Using the vector potential representation $\mathbf{B}=\nabla \times \mathbf{A}$ and the reality condition $\mathbf{B}_{-k}=\mathbf{B}_{k}^{*}$ of the Fourier modes (Matthaeus \& Goldstein 1982), $H_{m}$ can be represented as a superposition of the spectral magnetic helicity $H_{\mathrm{mk}}$ for each mode

$$
H_{m}=\frac{1}{2} \sum_{k} H_{\mathrm{mk}}=\frac{1}{2} \sum_{k}\left(A_{k} \cdot B_{k}^{*}\right)=\sum_{k} k \operatorname{Im}\left(A_{\mathrm{yk}}^{*} A_{\mathrm{zk}}\right) .
$$

Thus, by representing the magnetic field and vector potential in components in Equations (2) and (3), and after some algebraic manipulations, we can express $H_{\mathrm{mk}}$ in terms of the coefficients $\left(C_{k}^{L}, C_{k}^{R}\right)$ as

$$
H_{\mathrm{mk}}=\frac{1}{2 k}\left(\left|C_{k}^{L}\right|^{2}-\left|C_{k}^{R}\right|^{2}\right) .
$$

This result shows that for parallel propagation at least, the magnetic helicity spectrum is independent of the Fourier spectral phases and only depends on the Fourier amplitude coefficients. To the best of our knowledge, this aspect seems to be an unknown result for parallel propagating waves.

We can now define a normalized magnetic helicity for each Fourier mode in terms of the spectral energy density and spectral helicity as follows:

$$
\sigma_{\mathrm{mk}}=\frac{k H_{\mathrm{mk}}}{\left|B_{k}\right|^{2}}=\frac{\left(\left|C_{k}^{L}\right|^{2}-\left|C_{k}^{R}\right|^{2}\right)}{\left(\left|C_{k}^{L}\right|^{2}+\left|C_{k}^{R}\right|^{2}\right)}
$$

Therefore, the Fourier coefficients $\left(C_{k}^{L}, C_{k}^{R}\right)$ can be obtained using Equations (5) to (9) as

$$
\begin{gathered}
\left|C_{k}^{L}\right|^{2}=\left|B_{k}\right|^{2}\left(1+\sigma_{\mathrm{mk}}\right)=\frac{\eta^{2} B_{0}^{2}}{2}\left[\frac{k^{-\alpha}\left(1+\sigma_{\mathrm{mk}}\right)}{\sum_{k>0} k^{-\alpha}}\right] \\
\left|C_{k}^{R}\right|^{2}=\left|B_{k}\right|^{2}\left(1-\sigma_{\mathrm{mk}}\right)=\frac{\eta^{2} B_{0}^{2}}{2}\left[\frac{k^{-\alpha}\left(1-\sigma_{\mathrm{mk}}\right)}{\sum_{k>0} k^{-\alpha}}\right],
\end{gathered}
$$

where the power spectral energy density profile $\left|\boldsymbol{B}_{k}\right|^{2}$ has been defined as a power law and $\left|\sigma_{\mathrm{mk}}\right| \leqslant 1$ for each wave mode, where $\sigma_{\mathrm{mk}}= \pm 1$ represents wave modes with left- $(-)$ or righthanded $(+)$ circular structure. If $\left|\sigma_{\mathrm{mk}}\right|<1$, then the wave is elliptical with an admixture of both left- and right-handed structures. Once the Fourier coefficients have been determined from Equation (10), an inverse Fourier transform can be carried out to generate the magnetic fluctuations in the configuration space.

\subsection{Alfvénic Fluctuations}

Although the previous theoretical calculations define the magnitude of the Fourier coefficients, they are not representative of Alfvénic fluctuations by themselves. To characterize the type of the fluctuations, relating the magnetic fluctuations to the plasma medium in which they propagate is required. In the case of MHD fluctuations, this is simply carried out by solving the multi-fluid equations describing the mass and momentum conservation equations for a plasma of species $s$ together with Maxwell's equations. Linearizing these equations for purely parallel propagation, the transverse magnetic field and velocity fluctuations in a drifting plasma are related according to:

$$
\delta U_{s \perp k}^{ \pm}=-\left[\frac{\left(\omega / k-U_{\| s}\right)}{\left(1 \mp\left(\omega-k U_{\| s}\right) / \Omega_{s}\right)}\right] \frac{\delta B_{\perp k}^{ \pm}}{B_{0}} .
$$

Here we have defined the velocity and magnetic components in the usual rotating coordinate system (represented by the \pm superscripts) defined by $B_{k}^{-}=B_{\mathrm{yk}}-i B_{\mathrm{zk}}=$ $C_{k}^{L} \exp \left(i \varphi_{k}\right)$ and $B_{k}^{+}=B_{\mathrm{yk}}+i B_{\mathrm{zk}}=C_{k}^{R} \exp \left(i \psi_{k}\right)$, so the $\mp$ sign represents left- and right-handedness of the waves, respectively. $\Omega_{s}$ is the species cyclotron frequency, $U_{\| s}$ is the species parallel drift velocity and $\omega / k$ is the phase velocity of the fluctuations obtained from the dispersion relation $\omega=\omega(k)$. For example:

$$
D^{(L, R)}(\omega, k)=1-\sum_{s} \frac{\omega_{\mathrm{ps}}^{2}}{k^{2} c^{2}} \frac{\left[\left(\omega-k U_{\| s}\right) / \Omega_{s}\right]^{2}}{\left[1 \mp\left(\omega-k U_{\| s}\right) / \Omega_{s}\right]}=0 .
$$

Here the $\mp$ sign corresponds to left and right circular polarization for positive wave number $k$. Once the fluctuating velocities have been determined using (11) and (12), the normalized cross-helicity can be evaluated for each wave mode and for each species. The cross-helicity provides the direction of wave energy propagation. The normalized cross-helicity is defined as (Matthaeus \& Goldstein 1982)

$$
\sigma_{\mathrm{csk}}=\frac{\left(\delta \mathbf{U}_{\mathrm{sk}} \cdot \delta \mathbf{U}_{A k}\right)}{\left|\delta \mathbf{U}_{\mathrm{sk}}\right|^{2}+\left|\delta \mathbf{U}_{A k}\right|^{2}},
$$

where $\delta \mathbf{U}_{A k}$ represents the transverse magnetic field in units of the Alfvén speed in the Fourier domain. The sign of the crosshelicity spectrum gives the propagation direction of the wave energy. A negative sign corresponds to wave energy propagating parallel to the mean field, whereas a positive sign represents anti-parallel propagation. From the definition of the normalized magnetic helicity and cross-helicity, the polarization (in the plasma physics sense) can be determined. In the fluid limit, if the product of the normalized helicities is positive (i.e., $\sigma_{\mathrm{mk}} \cdot \sigma_{\mathrm{ck}}>0$ ) or negative (i.e., $\sigma_{\mathrm{mk}} \cdot \sigma_{\mathrm{ck}}<0$ ), then the fluctuations are left- or right-handed polarized waves in the plasma physics sense, respectively (Smith et al. 1984; Viñas et al. 1984; Goldstein et al. 1985). 

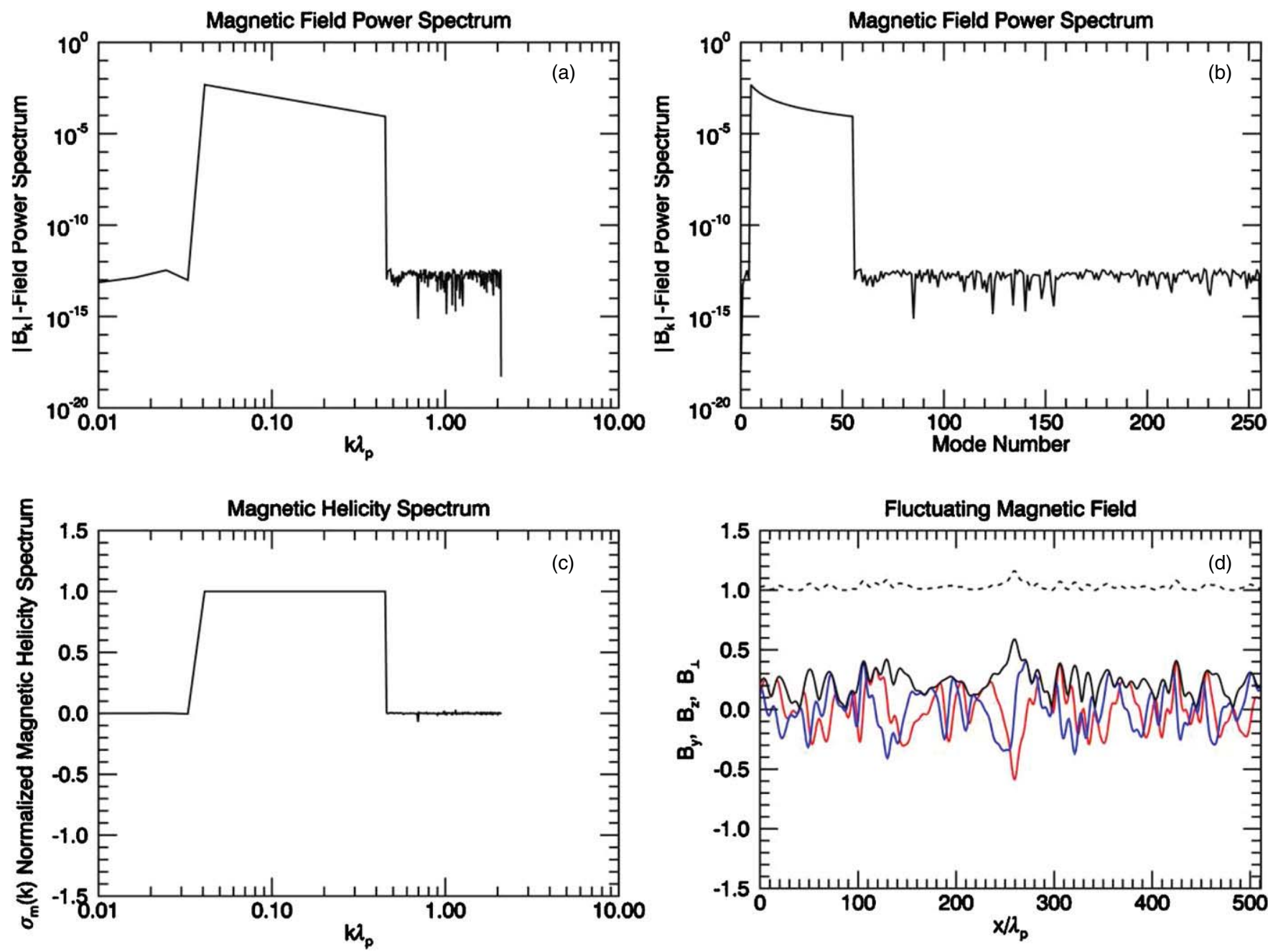

Figure 2. Power law magnetic field power spectrum $k^{-5 / 3}$, amplitude $\eta=0.25$, vs. normalized (a) wavenumber and (b) mode number. (c) Normalized magnetic helicity spectrum $\sigma_{\mathrm{mk}}$. (d) Magnetic field components in real space resulting from the power spectrum. Red, blue, solid black, and dashed black curves correspond to $\delta B_{y}, \delta B_{z},\left|\delta \mathbf{B}_{\perp}\right|$, and $|\delta \mathbf{B}|$, respectively. The field magnitude in (d) has been shifted for clarity.

(A color version of this figure is available in the online journal.)

\section{RESULTS}

We apply the developed theory for general type broadband spectra of magnetic field fluctuations to construct a spectrum of Alfvénic fluctuations propagating through an isotropic electrons, protons plasma with a 5\% alpha particles and no differential ion streaming. The solution of the dispersion relation (Equation (12)) for parallel wave propagation in such a plasma is shown in Figure 1. Electrons are included only as a neutralizing fluid background. We assumed a prescribed power-law power spectrum with spectral index $\alpha=5 / 3$, rms amplitude $\eta=0.25$, and $10^{-5} \%$ noise level as shown in Figure 2(a). We used a onedimensional grid of 512 cells of size 1.5 in units of the proton inertial length $\left(\lambda_{p}\right)$, and from all the 256 Fourier modes allowed by the grid, we selected a spectral range between modes 5 and 55 (see Figure 2(b)) that correspond to fluctuations with wave numbers between $k \lambda_{p} \sim 0.04$ and 0.45 . Using that particular spectrum, we reconstructed the magnetic field for a left-handed magnetic helicity $\left[\sigma_{\mathrm{mk}}=1\right.$, Figure 2(c)]. Thus, once we have selected the profile, amplitude, and helicity of the spectrum, we can uniquely determine the Fourier coefficients $\left(C_{k}^{L}, C_{k}^{R}\right)$ in Equation (10) and carry out an inverse Fourier transform to obtain the magnetic field in configuration space (Equation (1)).
In Figure 2(d), we show the resulting magnetic field components, perpendicular amplitude, and total amplitude for lefthanded Alfvénic fluctuations $\left(\sigma_{\mathrm{mk}}=1\right)$. In Figure 3, we show the self-consistent bulk velocity components and their corresponding cross-helicity spectra for protons and helium fluids. These results are obtained following the left-hand alpha Alfvén cyclotron branch on the dispersion relation (Figure 1, green line) for $k>0$. The results in these figures also indicate that the resultant plasma physics polarization of the spectrum is selfconsistent $\left(\sigma_{\mathrm{mk}} \cdot \sigma_{\mathrm{ck}}>0\right)$ with the left-handed branch selected in the solution to the dispersion relation (Equation (12)) and in Figure 1 (green line) for the reconstruction of the broad spectrum. Moreover, the magnitude of the cross-helicity spectrum is about $\sigma_{\mathrm{ck}}=1$, suggesting that the energy flow of these fluctuations is anti-parallel to the mean field. Both cross-helicity spectra for protons and helium show a decrease at the upper end of the spectra, which is due to a change in the dispersion curve as the wave vector increases and the frequency approaches the helium-cyclotron frequency. Although the reconstruction was estimated with an amplitude fluctuation of $\eta=0.25$, the resultant module of the magnetic field magnitude (Figure 2(d), dashed lines) is essentially constant, with a standard deviation of the magnitude fluctuation about its mean near 0.03. This 

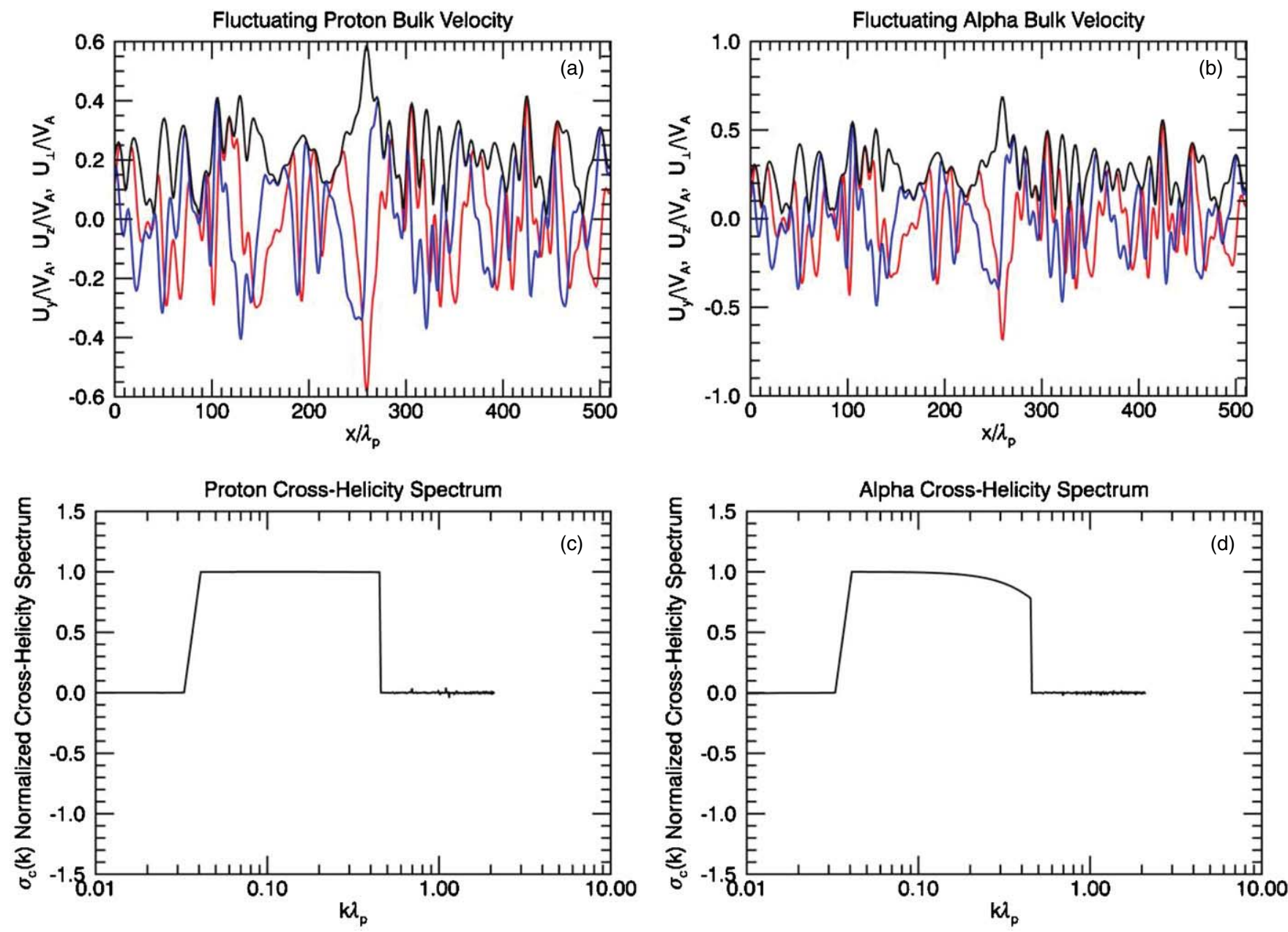

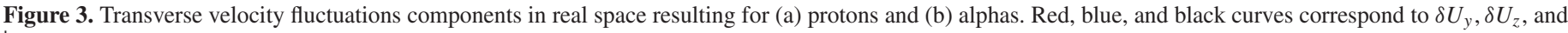
$\left|\delta \mathbf{U}_{\perp}\right|$, respectively. Cross-helicity spectrum for (c) protons and (d) alphas. All velocities are in units of the Alfvén speed.

(A color version of this figure is available in the online journal.)

result is also independent of the seed used in the random generator. This small fluctuation is consistent with almost constancy of the field magnitude expected for Alfvénic fluctuations observed in the solar wind (see Roberts 2012).

Similar results were also obtained in Figure 4 for the righthanded branch of the dispersion relation in Figure 1 (black line). Figures 3(a)-(d) shows the reconstructed spectrum of righthanded Alfvénic fluctuations assuming similar amplitudes as in the previous case, but with a broader spectral range extending slightly over the ion inertial length and for an initial magnetic helicity of $\sigma_{\mathrm{mk}}=-1$. Note the almost constant total magnetic field magnitude and consistent right-handed plasma physics polarization $\left(\sigma_{\mathrm{mk}} \cdot \sigma_{\mathrm{ck}}<0\right)$. Note also the appearance of higher-frequency (shorter-scale) fluctuations. Furthermore, the cross-helicity spectrum shows a significant decrease at shorter wavelengths since the spectrum is becoming more dispersive as the frequency approaches the alphas-cyclotron frequency. Similar results were obtained for the magnetic helicity spectrum by $\mathrm{He}$ et al. (2011) at shorter wavelengths. For scales above the ion-inertial length, Equations (11) and (13) may no longer be valid, thus the significance of the cross-helicity should be revised.

Finally, to illustrate the application of our model, we chose to represent and compare a similar time series of magnetic fluctuations as recently studied by Roberts (2012). Figure 5 shows the resulting magnetic fluctuations for a spectrum reconstructed with similar parameters as those used by Roberts (2012; see his Figure 4) in his observations. His spectrum is formed from a time series of about 4000 data points at $d t=0.125 \mathrm{~s}$, a maximum normalized rms amplitude of 1 , and a spectrum of $f^{-5 / 3}$. We use similar parameters, but in the $k$-domain, where we generated a spatial magnetic field series of 4096 data points, with $d x=0.125$, a maximum normalized $\mathrm{rms}$ amplitude of $\eta=1$, and a spectrum of $k^{-5 / 3}$. Since no information about the magnetic helicity spectrum was presented, we chose to generate the spectrum using a fixed magnetic helicity magnitude $\left|\sigma_{\mathrm{mk}}\right|=1$, but with a random sign fluctuation. The resulting magnetic fluctuations for $\eta=1$ are depicted in Figure 5(a), which shows the components, the transverse magnitude, and the total magnitude (shifted upward for clarity) of the magnetic field. An estimate of the standard deviation for the magnitude in Figure 5(a) yields 0.33 , which continues to be small even when the fluctuation amplitudes used are on the order of one $(\eta=1)$. A quick comparison of these "time series" with Figure 4 in Roberts (2012) shows that the magnetic fluctuations present sudden changes ("discontinuities") similar to those he observed. Furthermore, our results also depict Alfvénic fluctuations with essentially constant magnetic field magnitudes consistent with the results of Roberts (2012). Similar results are obtained increasing the fluctuation amplitude to $\eta=2$ in Figure 5(b). In this case, the 

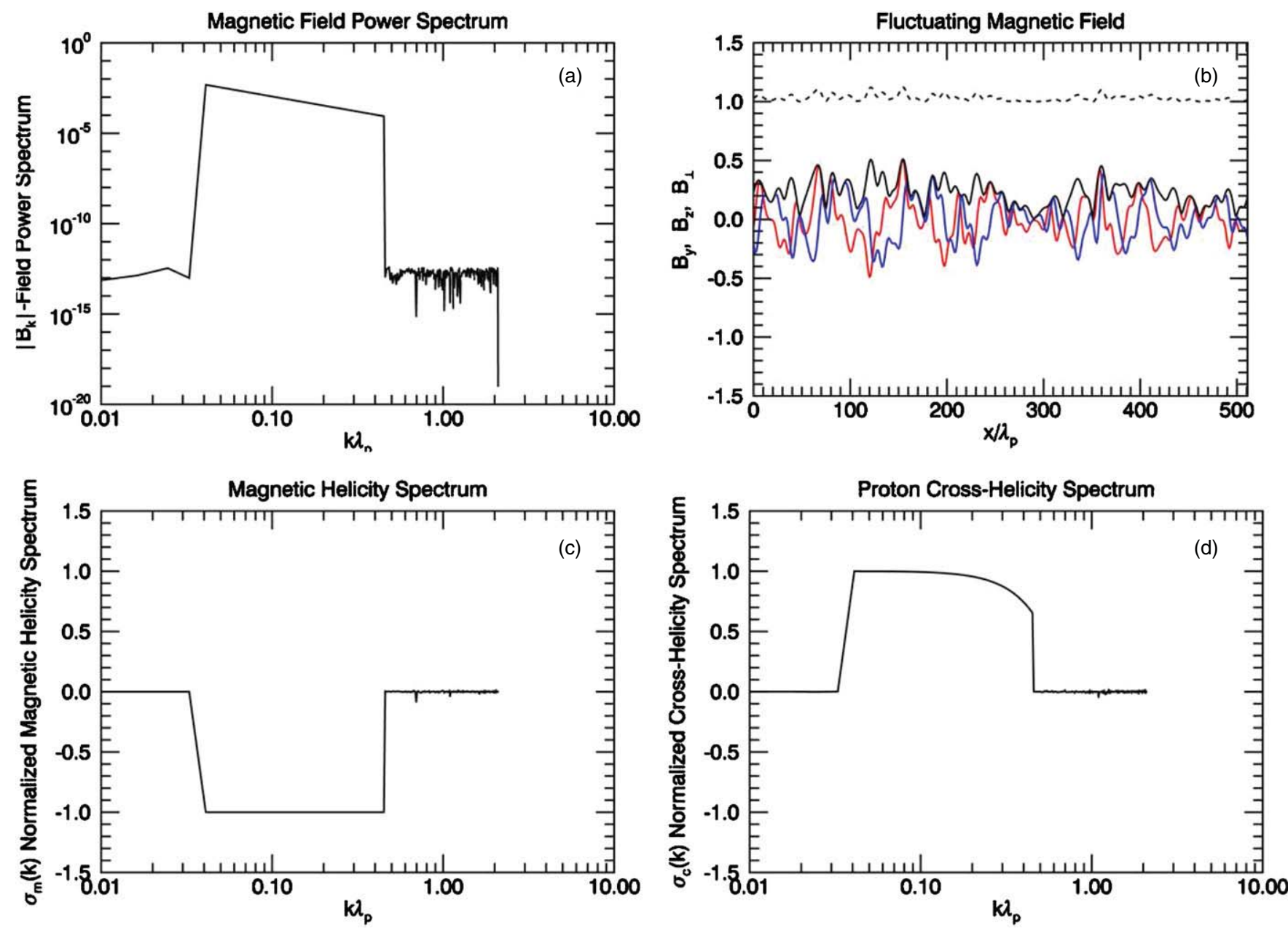

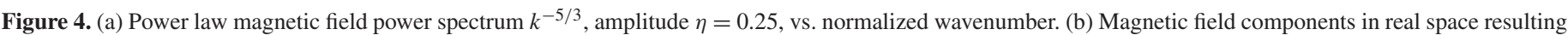

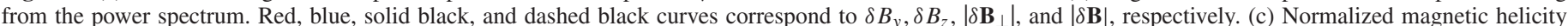
spectrum $\sigma_{\mathrm{mk}}$. (d) Corresponding proton cross-helicity spectrum.

(A color version of this figure is available in the online journal.)

standard deviation of the field magnitude fluctuation increases to about 0.83 , indicating that the constancy of the magnetic field magnitude breaks down as $\eta$ increases. Thus, for very large amplitudes, the reconstruction scheme presented here may require an optimized approach similar to that used by Roberts (2012) to account for the near constancy of the magnetic field magnitude. Nonetheless, the constraints introduced by the magnetic helicity and cross-helicity are also necessary in describing the Alfvénic fluctuations.

\section{DISCUSSION AND CONCLUSIONS}

We have described a new approach for the reconstruction of a broadband spectrum of parallel-propagating Alfvénic waves, including the self-consistent velocity fluctuations of the plasma. This physically motivated model constrained by observables allows us to initiate theoretical and simulation turbulence studies directed toward the understanding of finite amplitude waves in many scales of the solar wind. Starting from particular chosen parameters typical to those observed in the solar wind and solving the multifluid wave dispersion relation for parallel propagation, we have shown the reconstruction of magnetic and velocity fluctuations as well as the calculation of the crosshelicity needed to obtain the plasma physics polarization of fluctuations used in both observational and theoretical studies of plasmas.

Our results show rapid magnetic field changes despite the prescribed (fixed) spectral shape and sense of magnetic helicity. This result seems consistent with what Roberts (2012) called "discontinuities" as determined by his phase optimization approach. It is remarkable that even though we do not impose the constancy of the field magnitude, our theoretical model provides consistently similar results as those of Roberts (2012). It is clear that the magnetic field magnitude constancy in the reconstruction approach presented here breaks down as the amplitude of the fluctuation is increased well beyond $\eta \geqslant 2$. The usage of the energy conservation, magnetic helicity preservation, and crosshelicity imposes further constraints that seem to help reduce the standard deviations of the magnitude fluctuations for moderate amplitudes $(\eta \leqslant 1)$. This is independent of the random phases.

A generalization of the concepts presented here for the reconstruction of a two-dimensional case is being carried out at present, and should reveal interesting comparisons with simulation results for obliquely propagating fluctuations. The results presented here and motivated in combination with those of Roberts (2012), Malara \& Velli (1996), and Umeki \& Terasawa (1992) may lead us to a robust reconstruction and representation of the spectrum of Alfvénic fluctuations in the 
Fluctuating Magnetic Field
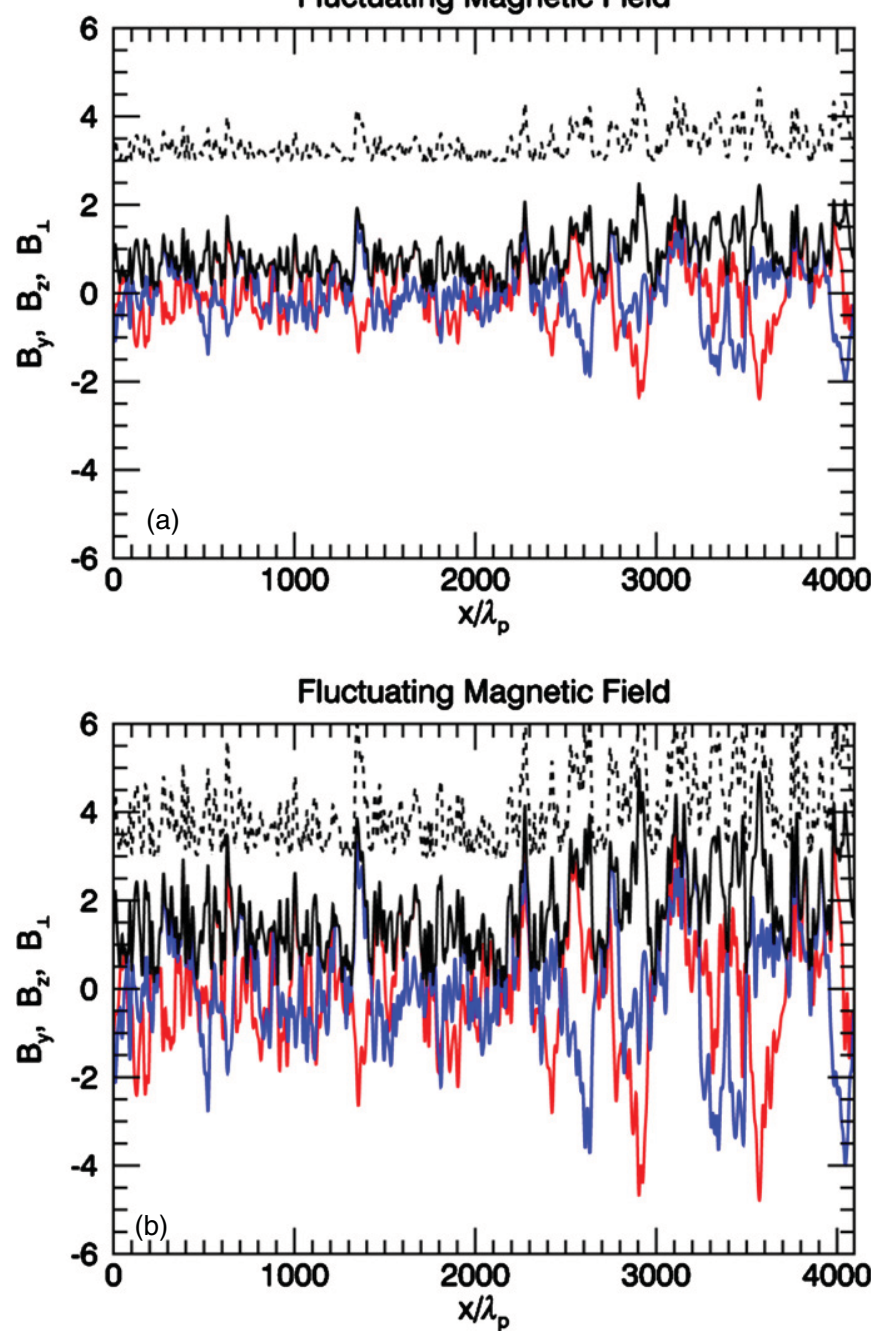

Figure 5. Magnetic field components in real space similar to Figure 4 in Roberts (2012). Red, blue, solid black, and dashed black curves correspond to $\delta B_{y}, \delta B_{z}$, $\left|\delta \mathbf{B}_{\perp}\right|$, and $|\delta \mathbf{B}|$, respectively, for (a) $\eta=1$ and (b) $\eta=2$.

(A color version of this figure is available in the online journal.)

solar wind. The model presented here provides an alternate approach to the reconstruction of a broadband spectrum of
Alfvénic fluctuations. These physically motivated theoretical models form the basis of initial conditions for many types of simulations that include nonlinear codes, from the MHD inertial range throughout the kinetic ions or electron scales, and provide a framework for more realistic simulations of plasma waves, solar wind turbulence, and the propagation of energetic particles in such fluctuating fields.

We thank Dr. Robert Wicks for providing critical suggestions and comments on this paper, and the NASA Wind/SWE program for the support of this research. We also thank the Comision Nacional de Ciencia y Tecnologia (CONICyT, Chile) for providing financial support for P.S.M.'s postdoctoral fellowship. Y.M. thanks the NASA Wind and CUA post-doctoral programs for their financial support. J.A.A. thanks FONDECYT (No. 1110880) for providing financial support.

\section{REFERENCES}

Agim, Y., Viñas, A. F., \& Goldstein, M. L. 1995, JGR, 100, 17081 Araneda, J. A., Marsch, E., \& Viñas, A. F. 2008, PhRvL, 100, 125003 Barnes, A. 1981, JGR, 86, 7498

Belcher, J. W., \& Davis, L., Jr. 1971, JGR, 76, 3534

Bruno, R., \& Carbone, V. 2013, LRSP, 10, 2

Cohen, R. H. 1975, JGR, 80, 3678

Cohen, R. H., \& Dewar, R. L. 1974, JGR, 79, 4174

Ghosh, S., Viñas, A. F., \& Goldstein, M. L. 1994, JGR, 99, 19289

Goldstein, M. L., Wong, H. K., Viñas, A. F., \& Smith, C. W. 1985, JGR, 90, 302 He, J., Marsch, E., Tu, C., Yao, S., \& Tian, H. 2011, ApJ, 731, 85

He, J., Tu, C., Marsch, E., \& Yao, S. 2012, ApJ, 749, 86

Hoshino, M., \& Goldstein, M. L. 1989, PhFlB, 1, 1405

Malara, F., \& Velli, M. 1996, PhPl, 3, 4427

Maneva, Y. G., Viñas, A. F., \& Ofman, L. 2013, JGR, 118, 2842

Marsch, E. 2006, LRSP, 3, 1

Matteini, L., Landi, S., Del Zanna, L., Velli, M., \& Hellinger, P. 2010, GeoRL, 37, L20101

Matteini, L., Landi, S., Velli, M., \& Hellinger, P. 2010, JGR, 115, A09106

Matthaeus, W. H., \& Goldstein, M. L. 1982, JGR, 87, 6011

Moya, P. S., Viñas, A. F., Muñoz, V., \& Valdivia, J. A. 2012, AnGeo, 30, 1361

Podesta, J. J., \& Gary, S. P. 2011, ApJ, 734, 15

Roberts, D. A. 2012, PhRvL, 109, 231102

Smith, C. W., Goldstein, M. L., Matthaeus, W. H., \& Viñas, A. F. 1984, JGR, 89,9159

Umeki, H., \& Terasawa, T. 1992, JGR, 97, 3113

Viñas, A. F., Goldstein, M. L., \& Acuña, M. H. 1984, JGR, 89, 3762

Wicks, R. T., Forman, M. A., Horbury, T. S., \& Oughton, S. 2012, ApJ, 746,103 